\title{
Clinical characteristics of drug-induced liver injury and related risk factors
}

\author{
REN-JIE LU ${ }^{1}$, YAN ZHANG ${ }^{2}$, FENG-LEI TANG ${ }^{1}$, ZHONG-WEI ZHENG ${ }^{2}$, ZHENG-DA FAN $^{1}$, \\ SHAN-MEI ZHU ${ }^{1}$, XIAN-FENG QIAN ${ }^{2}$ and NA-NA LIU ${ }^{2}$ \\ Departments of ${ }^{1}$ Pharmacy and ${ }^{2}$ Digestive Diseases, The Third People's Hospital \\ of Changzhou, Changzhou, Jiangsu 213000, P.R. China
}

Received February 11, 2016; Accepted August 19, 2016

DOI: $10.3892 /$ etm.2016.3627

\begin{abstract}
Drug-induced liver injury (DILI) is often undiagnosed or misdiagnosed clinically because of diagnostic difficulties caused by lack of laboratory-specific serological markers. In this study, we comprehensively assessed the clinical characteristics, laboratory indices, hepatotoxic drugs, risk factors and outcomes concerning DILI, and explored the similarities in mechanisms between Chinese and Western drug-induced DILI. Patients with a first diagnosis of DILI and a Roussel Uclaf Causality Assessment Method (RUCAM) score $>3$ points were enrolled for systematic retrospective study. Their clinical characteristics, clinical classification, risk factors, laboratory indices, hepatotoxic drugs and outcomes were analyzed. Cholestatic patients had the highest alkaline phosphatase (ALP) and prothrombin time activity (PTA) levels $(\mathrm{P}<0.05)$. Patients with medication time $\geq 30$ days had significantly higher positive rate of autoantibodies than those with medication time $<30$ days. Odds ratio values for DILI-related factors such as hepatobiliary diseases, immune dysfunction, diabetes, hypertension, chronic alcohol consumption and age $\geq 45$ years were 6.552 , 6.130, 3.774, 2.801, 2.002 and 1.838, respectively. Pathogeneses of Chinese and Western drug-induced DILI may be substantially the same. DILI accompanied with autoantibody positivity may indicate severe liver injury outcome. Hepatobiliary diseases, diabetes and hypertension are likely to increase drug susceptibility, and more prone to cause liver injury.
\end{abstract}

\section{Introduction}

The liver is the principle organ for drug metabolism and transformation. It not only processes the three major metabolites but is also an important organ for drugs and drug metabolism. Drug-induced liver injury (DILI) refers to a disease caused by

Correspondence to: Dr Zhong-Wei Zheng, Department of Digestive Diseases, Third People's Hospital, Changzhou, Jiangsu 213000, P.R. China

E-mail: qsf876267811@163.com

Key words: drug-induced liver injury, clinical feature, risk factor, pathogenesis hepatotoxic injury induced by drugs or their metabolites or due to hepatic hypersensitivity to drugs and their metabolites during medication. In recent years, with the extensive use of drugs and the accelerated research of novel drugs, the different types of clinical drugs have been continuously increasing and the incidence of DILI has been rising year by year. According to World Health Organization (WHO) statistics, DILI has risen as the 5th leading cause of death worldwide. Approximately $50 \%$ of liver failure patients receiving liver transplantation every year have drug-induced liver failure, which prompts the need for artificial liver and liver transplantation and even causes death $(1,2)$. However, the disease is often undiagnosed or misdiagnosed clinically because of diagnostic difficulties caused by lack of laboratory specific serological markers.

There are more than 1,000 types of drugs that can lead to DILI, of which common ones include Chinese herbal drugs (Polygonum multiflorum, Cassia seed, Mentha, Rheum and Tripterygium wilfordii), antituberculotics [such as isoniazid (INH), rifampicin (RFP), pyrazinamide (PZA) and sodium p-aminosalicylate], antipyretic analgesics [such as acetaminophen (APAP), aspirin and phenylbutazone], antibiotics (such as macrolides, penicillins and quinolones), and antineoplastics. Currently, it is believed that the pathogenesis of DILI may be through three major pathways: i) direct cellular stress induced by drugs and their metabolites (intrinsic pathway); ii) direct damage to mitochondrial function; iii) activation of specific immune responses (extrinsic pathway), thereby changing mitochondrial permeability, and ultimately leading to apoptosis (3). In recent years, research concerning the pathogenesis of DILI has mainly focused on the following aspects: genetics, e.g., cytochrome P450, nucleoside diphosphate glucuronosyltransferases, $\mathrm{N}$-acetyltransferase 2 and glutathione $\mathrm{S}$-transferase; human leukocyte antigens, e.g., specific human leukocyte antigen (HLA) genotype, HLA DRP1-1501 (predictor for hepatotoxicity of amoxicillin/clavulanate) and HLA UGT1A1 (predictor for hepatotoxicity of pazopanib); mitochondrial oxidative stress-related enzymes; and transmembrane transporters. Recently, some studies have found that Fra-1 protein plays an important hepatoprotective role, which can activate the expression of anti-toxin genes as well. APAP overdose can cause significantly increased fibroblast growth factor 21 . This phenomenon may be a compensatory mechanism preventing DILI, which can enhance the hepatic antioxidant capacity 
mediated by peroxisome proliferator-activated receptor $\gamma$ co-activator $1 \alpha /$ nuclear factor E2-related factor. Immune injury is another important pathway to DILI. Drugs can cause liver cell necrosis as foreign antigens by activating the immune system and then mediating corresponding injuries. According to recent studies, microRNA (miR)-122, as a highly liver-specific miR, can be used as a molecular marker of hepatocellular injury (4), whose specificity and sensitivity are significantly higher than alanine aminotransferase (ALT) in the early diagnosis of APAP-induced DILI (5). Some pathological findings may be relatively specific. The common histopathological changes in the liver may include ballooning changes or microvesicular steatosis, eosinophil infiltration, epithelial cell granuloma and cholestasis, but pathological evidence makes sense only in combination with medical history and other clinical data.

DILI is classified into two types, one type is associated with the toxicity of drugs, which causes liver injury by directly injuring liver cells or interfering with biliary excretion, and possesses predictable, dose-dependent and reproducible features. The other type, also known as idiosyncratic DILI, is associated with the idiosyncrasy of drug receivers such as genetic and metabolic factors, which is often accompanied by allergic and autoimmune reactions. It has the following characteristics: i) dose-independency, unpredictability, occurs only in certain people or groups, or familial aggregation; ii) can be accompanied by fever, joint pain, rashes and other extrahepatic manifestations; and iii) eosinophilia, positive autoantibodies and elevated immunoglobulins (Igs). There exist immune-mediators in idiosyncratic DILI, which may exhibit varying degrees of elevation along with positivity of autoantibodies, such as anti-nuclear and anti-smooth muscle antibodies; immunoglobulin elevation is often misdiagnosed as autoimmune hepatitis or other autoimmune liver diseases. In recent years, with the improvement of autoantibody detection technologies, the diagnosis, differential diagnosis and treatment of liver dysfunction patients with positive autoantibodies, particularly high-titer autoantibodies have gained increasing attention.

Clinical characteristics are still an important basis for diagnosing drug-induced liver diseases, and current understanding of these characteristics requires further improvement. Thus, in the present study, DILI patients with a Roussel Uclaf Causality Assessment Method (RUCAM) score $>3$ points were enrolled for the systematic retrospective study, and their clinical characteristics, clinical classification, risk factors, laboratory indices, hepatotoxic drugs and prognosis were analyzed, which are of great clinical value for comprehensively understanding DILI.

\section{Patients and methods}

Patient selection. Retrospectively, 513 patients who were hospitalized with a first clinical diagnosis of DILI from January 2011 to December 2014 were selected for this study. Based on the information of the hospitalized patients such as clinical symptoms, laboratory indices, underlying diseases, hepatotoxic drugs, liver histopathology and efficacy after withdrawal, the association between medication and liver injury was assessed comprehensively with RUCAM as the standard quantitative assessment system (6) to include 424 patients, and exclude 89 patients.

Inclusion diagnosis. Currently, the RUCAM scale is a universally recognized method for evaluating the hepatotoxicity of drugs. Using the RUCAM system, association between medication and liver injury was assessed comprehensively, and quantitative scoring was performed for each case. Assessment content was comprised of 7 items, time from drug intake to onset, liver function recovery after withdrawal, risk factors, simultaneous medication, exclusion of other factors, previous liver injury by drugs and reactions to re-medication. The above items were scored one by one as follows: i) $\leq 0$, rule out DILI; ii) 1-2 points, unlikely; iii) 3-5 points, likely; iv) 6-8 points, very likely; and v) $>8$ points, highly likely. Patients with scores $>3$ points were included: all 'likely', 'very likely' and 'highly likely' cases.

Exclusion diagnoses. Exclusion criteria included recent hepatitis A virus (HAV) infection (anti-HAV-IgM positive), HBV infection (anti-HBc-IgM positive), HCV infection (anti-HCV positive) and other evidences of hepatitis virus infection; biliary obstruction (confirmed by B ultrasound); excessive alcohol [aspartate aminotransferase (AST)/ALT ratio $\geq 2$ ]; acute hypertension (especially those associated with severe heart diseases); cytomegalovirus, Epstein-Barr virus or herpes virus infection; autoimmune liver diseases, alcoholic liver diseases, genetic metabolic liver diseases, liver injury induced by hemodynamic abnormalities; or other severe disease complications.

Detection instruments. Detection instruments included automatic biochemistry analyzer (AU2700, Olympus, Tokyo, Japan); automated microplate reader (680; Bio-Rad, Berkeley, CA, USA); automated immunoblot processor (EURO Blot Master II; Euroimmun AG, Lübeck, Germany); hematology analyzer (HMX, 5Diff; Beckman Coulter, Miami, FL, USA); automatic coagulation analyzer (STA-R; Diagnostica Stago S.A.S, Asnières-sur-Seine, France); real-time polymerase chain reaction system (7500; Applied Biosystems, Foster City, CA, USA); genetic analyzer (310; Applied Biosystems); time-resolved fluoroimmunoassay (TRFIA) system (AutoDELFIA-1235; Perkin-Elmer, Waltham, MA, USA USA).

Laboratory indices. Laboratory indices included alanine aminotransferase (ALT; 9-50 U/1), aspartate aminotransferase (AST; 15 40 U/1), alkaline phosphatase (ALP, 45-125 U/1), serum total bilirubin (TBiL, 3.4 22 $\mu \mathrm{mol} / \mathrm{l}$ ), prothrombin time (PT, 11-15 sec), prothrombin activity (PTA, 75-160\%), international normalized ratio (INR, 0.8-1.5), albumin (ALB; 40-55 g/l), total bile acid (TBA; 0-15 $\mu \mathrm{mol} / \mathrm{l}$ ), cholinesterase (ChE, 3,700-13,200 IU/1), anti-nuclear antibodies, anti-smooth muscle antibodies, anti-mitochondrial antibodies, as well as HAV, HBV, HCB, HDV and HEV antibodies.

Prognostic criteria. i) Cure: during hospitalization after treatment, clinical symptoms disappeared, and liver function returned to normal. ii) Improvement: during hospitalization after treatment, clinical symptoms improved markedly, and liver function indices improved compared with these indices 
Table I. Associations between clinical types and liver function-related indices in the DILI patients.

\begin{tabular}{lccccc}
\hline Clinical data & $\begin{array}{c}\text { Total, } \\
\mathrm{n}=424\end{array}$ & $\begin{array}{c}\text { Hepatocellular type, } \\
\mathrm{n}=239(56.4 \%)\end{array}$ & $\begin{array}{c}\text { Cholestatic type, } \\
\mathrm{n}=101(23.8 \%)\end{array}$ & $\begin{array}{c}\text { Mixed type, } \\
\mathrm{n}=84(19.8 \%)\end{array}$ & P-value \\
\hline Age, years & $47.9 \pm 15.2$ & $47.2 \pm 14.3$ & $51.8 \pm 18.9$ & $50.7 \pm 19.0$ & 0.388 \\
Male/female, cases & $155 / 269$ & $86 / 121$ & $30 / 71$ & $39 / 75$ & 0.882 \\
Length of stay, days & $29.4 \pm 18.4$ & $29.4 \pm 19.0$ & $29.9 \pm 16.1$ & $29.6 \pm 16.6$ & 0.993 \\
DILI duration, days & $16.2 \pm 12.2$ & $16.6 \pm 11.9$ & $13.9 \pm 13.6$ & $14.4 \pm 13.5$ & 0.866 \\
Medication time, days & $72.6 \pm 62.1$ & $77.6 \pm 65.4$ & $46.6 \pm 40.1$ & $47.6 \pm 41.3$ & 0.198 \\
Clinical indices & & & & \\
on admission & & & & & \\
ALT, U/l & $591.2 \pm 458.9$ & $608.4 \pm 475.8$ & $498.1 \pm 374.0$ & $525.6 \pm 370.0$ & 0.560 \\
AST, U/l & $349.1 \pm 341.0$ & $366.1 \pm 352.8$ & $273.3 \pm 263.5$ & $277.3 \pm 277.1$ & 0.363 \\
ALP, U/l & $208.5 \pm 146.4$ & $165.2 \pm 104.9$ & $415.6 \pm 149.8$ & $402.9 \pm 145.9$ & 0.000 \\
TBiL, $\mu$ mol/1 & $83.1 \pm 64.1$ & $77.6 \pm 65.3$ & $85.2 \pm 68.4$ & $89.8 \pm 68.2$ & 0.397 \\
PT, sec & $13.45 \pm 1.88$ & $13.57 \pm 1.96$ & $12.84 \pm 1.37$ & $12.91 \pm 1.39$ & 0.176 \\
PTA, \% & $97.11 \pm 23.77$ & $95.09 \pm 23.24$ & $114.65 \pm 16.26$ & $105.8 \pm 24.86$ & $0.002^{\mathrm{a}}$ \\
INR & $1.02 \pm 0.20$ & $1.04 \pm 0.21$ & $0.96 \pm 0.13$ & $0.97 \pm 0.13$ & 0.186 \\
Clinical indices & & & & & \\
at discharge & & & & & \\
ALT, U/l & & & & & \\
AST, U/l & $35.83 \pm 31.29$ & $28.63 \pm 19.45$ & $29.67 \pm 19.67$ & $37.27 \pm 33.19$ & 0.442 \\
ALP, U/l & $28.48 \pm 15.36$ & $26.06 \pm 12.18$ & $26.33 \pm 12.56$ & $28.97 \pm 15.96$ & 0.661 \\
TBiL, $\mu$ mol/1 & $106.34 \pm 55.07$ & $96.47 \pm 46.60$ & $150.81 \pm 68.29$ & $153.67 \pm 69.69$ & $0.000^{\text {a }}$ \\
PT, sec & $17.68 \pm 9.52$ & $17.29 \pm 9.18$ & $19.13 \pm 11.13$ & $19.87 \pm 11.12$ & 0.497 \\
PTA, \% & $13.49 \pm 1.33$ & $13.43 \pm 1.43$ & $13.00 \pm 0.77$ & $14.32 \pm 1.72$ & 0.319 \\
INR & $90.52 \pm 16.45$ & $86.66 \pm 13.77$ & $99.62 \pm 12.51$ & $82.68 \pm 22.32$ & 0.225 \\
\hline C & $1.07 \pm 0.14$ & $1.09 \pm 0.43$ & $1.01 \pm 0.81$ & $1.12 \pm 0.19$ & 0.458 \\
\hline
\end{tabular}

Compared with the hepatocellular and mixed type patients, ALP and PTA levels were the highest for cholestatic patients, ${ }^{a} \mathrm{P}<0.05$. DILI, drug-induced liver injury; ALT, alanine aminotransferase; AST, aspartate aminotransferase; ALP, alkaline phosphatase; TBiL, total bilirubin; PT, prothrombin time; PTA, prothrombin time activity; INR, international normalized ratio.

before treatment which were lower than 2 times the upper limits of normal; additionally, TBiL is normal. iii) Non-cure: during hospitalization after treatment, signs and symptoms failed to improve, and improvement of liver function was unobvious or more abnormal than on admission. iv) Death: during hospitalization, patients were unresponsive to treatment and died. Cured and improved patients constituted the effective group, whereas non-cured and dead patients constituted the ineffective group.

Statistical analysis. Measurement data are expressed as the mean \pm standard deviation, and were compared by t-test. Comparison of enumeration data between groups was performed by $\chi^{2}$ test, while pairwise comparison was carried out by Ridit test. Logistic regression was used to analyze the risk factors. Data were processed using SPSS 19.0 package (SPSS, Inc., Chicago, IL, USA).

\section{Results}

General information. Among all the 424 DILI patients included, 155 were males $(36.6 \%)$ and 269 were females
(63.4\%), with a male to female ratio of approximately $1: 1.43$. The mean patient age was $47.9 \pm 15.2$ years, and the mean length of stay was $29.4 \pm 18.4$ days. DILI duration was $16.2 \pm 12.2$ days and medication time was $72.6 \pm 62.1$ days. Based on the demarcation age of 55 years as in the RUCAM scale, the number of patients aged $\geq 55$ years totaled 162 (38.2\%), whereas those aged $<55$ years totaled $262(61.8 \%)$. In total, 163 patients (38.4\%) had originally been suffering from hepatobiliary diseases, while 235 patients $(55.4 \%)$ had underlying diseases.

Clinical symptoms. Patients mainly presented clinical symptoms such as weakness, poor appetite, yellowed skin, icteric sclera, dark urine, nausea, abdominal discomfort, fever, itchy skin, ascites and rash on admission. A total of 378 patients $(89.2 \%)$ manifested clinical symptoms, which in descending order of the number of symptomatic patients were weakness (288 patients, $67.9 \%$ ), poor appetite (270 patients, 63.7\%), dark urine (226 patients, $53.3 \%$ ), icteric sclera (172 patients, $40.6 \%$ ), yellowed skin (166 patients, 39.2\%), abdominal discomfort (132 patients, $31.1 \%$ ), nausea (102 patients, $24.1 \%$ ), itchy skin (44 patients, $10.4 \%$ ), fever (18 patients, $4.2 \%$ ), rash (16 patients, 
$3.8 \%$ ) and ascites (14 patients, 3.3\%). Meanwhile, 46 patients $(10.8 \%)$ showed no obvious clinical symptoms. Clinical symptoms of DILI with incidences $\geq 50 \%$ were weakness, poor appetite and dark urine in descending order.

Comparison of clinical types. Patients were grouped based on the laboratory indices of liver dysfunction on admission into hepatocellular type (239 patients, $56.4 \%$ ), cholestatic type (101 patients, 23.8\%) and mixed type (84 patients, 19.8\%). No significant difference was found in regards to the length of stay, degree of liver injury, clinical symptoms and partial laboratory indices (ALT, AST, TBiL, PT, INR) between the three groups of patients $(\mathrm{P}>0.05)$. Cholestatic patients had the

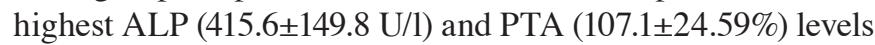
$(\mathrm{P}<0.05$; Table I).

\section{Autoantibody-positive rate in DILI patients}

Association between the autoantibody-positive rate and gender distribution. Among 155 (36.6\%) male patients, $89(57.4 \%)$ were autoantibody-positive and $66(42.6 \%)$ were autoantibody-negative. Among 269 (63.4\%) female patients, $162(60.2 \%)$ were autoantibody-positive and 107 (39.8\%) were autoantibody-negative. Autoantibody positive rate of male DILI patients was similar to the female patients, showing no significant difference (Table II).

Association between the autoantibody-positive rate and age distribution. Based on the demarcation age of 55 years as in the RUCAM scale, number of patients aged $\geq 55$ years totaled $162(38.2 \%)$, whereas those aged $<55$ years totaled $262(61.8 \%)$. For age $\geq 55$ years group, $89(54.9 \%)$ patients were autoantibody-positive and $73(45.1 \%)$ patients were autoantibody-negative. For age $<55$ years group, $147(56.1 \%)$ patients were autoantibody-positive and 115 (43.9\%) patients were autoantibody-negative. Comparison of autoantibody positive rate between age $\geq 55$ years and age $<55$ years groups found no significant difference (Table II).

Association between the autoantibody-positive rate and medication time. Patients were divided into two groups according to medication time, $\geq 30$ day group (266 patients, $62.7 \%$ ) and $<30$ day group (158 patients, $37.3 \%$ ). For the $\geq 30$ day group, $183(68.9 \%)$ patients were autoantibody-positive and $83(31.1 \%)$ patients were autoantibody-negative, while for the $<30$ days group, $63(39.9 \%)$ patients were autoantibody-positive and $95(60.1 \%)$ patients were autoantibody-negative. These results revealed a significantly higher autoantibody-positive rate in the medication time $\geq 30$ day group than the medication time $<30$ day group (Table II).

Association between the autoantibody-positive rate and CHILD classification. All DILI cases were subjected to CHILD classification, of which class A cases totaled 116 (27.4\%), class B cases totaled 223 (52.6\%), and class C cases totaled $85(20.0 \%)$. For patients with class A DILI, $59(50.8 \%)$ were autoantibody-positive and 57 (49.1\%) were autoantibody-negative; for patients with class B DILI, $140(62.8 \%)$ were autoantibody-positive and 83 (37.2\%) were autoantibody-negative; while for those with class C DILI, $61(72.8 \%)$ were autoantibody-positive and 24 (28.2\%) were

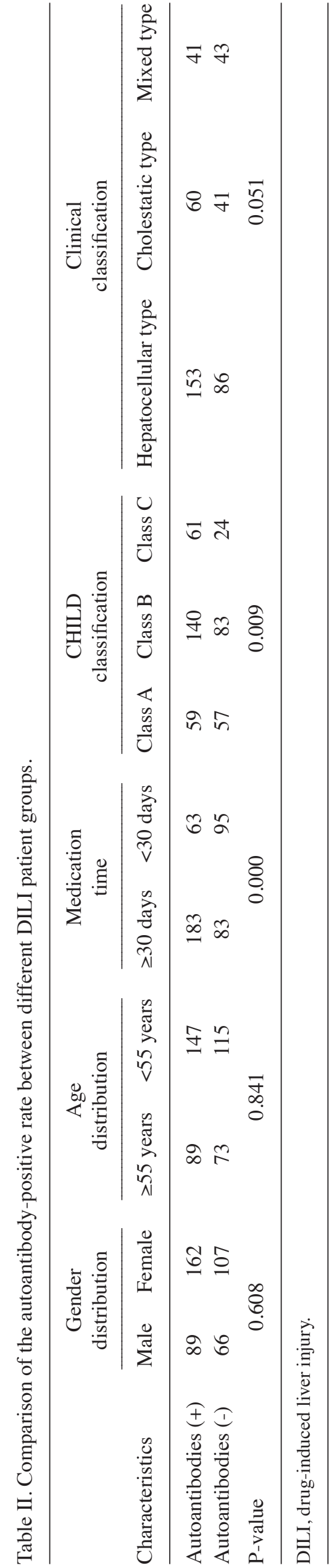


Table III. Comparison of clinical symptoms and laboratory indices between Chinese and Western drug-induced DILI groups.

\begin{tabular}{|c|c|c|c|}
\hline Group & Western drug group, $\mathrm{n}=172(59.4 \%)$ & Chinese drug group, $n=252(60.6 \%)$ & P-value \\
\hline Average age, years & $45.05 \pm 16.78$ & $49.74 \pm 12.86$ & 0.290 \\
\hline Length of stay, days & $29.81 \pm 14.37$ & $29.02 \pm 23.47$ & 0.791 \\
\hline DILI duration, days & $11.86 \pm 10.58$ & $22.21 \pm 15.87$ & 0.119 \\
\hline Medication time, days & $72.6 \pm 62.1$ & $77.7 \pm 67.1$ & 0.544 \\
\hline \multicolumn{4}{|l|}{ Clinical symptoms, $\mathrm{n}$} \\
\hline Weakness & 128 & 196 & 0.779 \\
\hline Poor appetite & 115 & 190 & 0.753 \\
\hline Dark urine & 84 & 172 & $0.020^{\mathrm{a}}$ \\
\hline \multicolumn{4}{|l|}{$\begin{array}{l}\text { Laboratory indices } \\
\text { on admission }\end{array}$} \\
\hline ALT, U/1 & $474.98 \pm 439.82$ & $521.02 \pm 456.02$ & 0.232 \\
\hline AST, U/1 & $302.47 \pm 256.01$ & $349.98 \pm 315.14$ & 0.303 \\
\hline ALP, U/1 & $159.28 \pm 114.52$ & $163.32 \pm 107.08$ & 0.802 \\
\hline $\mathrm{TBiL}, \mu \mathrm{mol} / \mathrm{l}$ & $71.39 \pm 60.18$ & $82.46 \pm 62.60$ & 0.833 \\
\hline PT, sec & $13.74 \pm 2.00$ & $13.64 \pm 2.55$ & 0.750 \\
\hline PTA, \% & $95.13 \pm 24.18$ & $95.43 \pm 22.55$ & 0.930 \\
\hline INR & $1.0426 \pm 0.20$ & $1.044 \pm 0.28$ & 0.959 \\
\hline \multicolumn{4}{|l|}{$\begin{array}{l}\text { Laboratory indices } \\
\text { at discharge }\end{array}$} \\
\hline ALT, U/1 & $46.76 \pm 23.80$ & $44.92 \pm 26.47$ & 0.722 \\
\hline AST, U/1 & $38.69 \pm 9.41$ & $34.38 \pm 5.22$ & 0.682 \\
\hline ALP, U/1 & $95.46 \pm 42.46$ & $99.97 \pm 50.64$ & 0.518 \\
\hline $\mathrm{TBiL}, \mu \mathrm{mol} / \mathrm{l}$ & $23.33 \pm 5.04$ & $21.04 \pm 4.30$ & 0.744 \\
\hline PT, sec & $15.67 \pm 1.01$ & $15.50 \pm 1.37$ & 0.359 \\
\hline PTA, \% & $80.83 \pm 7.56$ & $82.87 \pm 11.46$ & 0.169 \\
\hline INR & $1.07 \pm 0.64$ & $1.05 \pm 0.79$ & 0.146 \\
\hline
\end{tabular}

Compared to the Western drug group, dark urine symptom was more prevalent in the Chinese drug group, ${ }^{\text {a }}<0.05$. DILI, drug-induced liver injury; ALT, alanine aminotransferase; AST, aspartate aminotransferase; ALP, alkaline phosphatase; TBiL, total bilirubin; PTA, prothrombin time activity; INR, international normalized ratio.

autoantibody-negative. These results suggested that the autoantibody-positive rate rose gradually with the elevated CHILD score, showing significant differences (Table II).

Association between the autoantibody positive rate and clinical classification. According to the international clinical classification criteria, DILI was classified into hepatocellular type (239 patients, 56.4\%), cholestatic type (101 patients, $23.8 \%$ ) and mixed type (84 patients, 19.8\%). For hepatocellular patients, $153(64.0 \%)$ were autoantibody-positive and 86 $(36.0 \%)$ were autoantibody-negative. For cholestatic patients, $60(59.4 \%)$ were autoantibody-positive and 41 (40.6\%) were autoantibody-negative; while for mixed type patients, $41(48.8 \%)$ were autoantibody-positive and 43 (51.2\%) were autoantibody-negative. Autoantibody positive rate was slightly higher for the hepatocellular type than the cholestatic and mixed types, but the differences were not statistically significant (Table II).

Differences between Chinese and Western drug-induced DILI. Ranking of the number of patients with DILI-induced by different drugs found that Chinese drugs [decoctions, patent drugs, pastes and traditional Chinese medicine (TCM) health products] were responsible for the highest incidence of DILI (252 patients, $59.4 \%$ ), followed by antituberculotics (72 patients, $17.0 \%$ ), antipsychotics (27 patients, 6.37\%), non-steroidal anti-inflammatory drugs (NSAIDs) (17 patients, 4.01\%), antineoplastics (13 patients, 3.07\%), antithyroids (11 patients, $2.59 \%$ ), antibiotics (11 patients, $2.59 \%)$, antihypertensives (8 patients, $1.89 \%$ ), antidiabetics (6 patients, $1.42 \%$ ), antilipemics (3 patients, $0.70 \%$ ) and other drug types (6 patients, $1.42 \%$ ).

Distribution of Chinese drug-induced DILI. Chinese drugs were ranked for frequency of DILI induction, which were decoctions, patent drugs, pastes, and TCM healthcare products in a descending order by form. In terms of ingredients, the top three were Polygonum multiflorum (17 patients, 4.01\%), Tripterygium wilfordii (11 patients, $2.59 \%$ ) and Sedum aizoon (8 patients, $1.89 \%$ ). By usage, the rankings were treatment for hair loss (47 patients, 17.3\%), psoriasis (33 patients, 12.2\%), osteoarthrosis (32 patients, $11.8 \%$ ) and health care (29 patients, $10.7 \%$ ). 
Distribution of Western drug-induced DILI. Western drugs were ranked by frequency of DILI induction, which found the top 5 types to be antituberculotics (72 patients, $17.0 \%$ ), antipsychotics (27 patients, 6.37\%), NSAIDs (17 patients, $4.01 \%$ ), antineoplastics (13 patients, 3.07\%) and antithyroids (11 patients, $2.59 \%$ ). DILI-inducing antituberculotics included INH, RFP, PZA and ethambutol (EMB), mostly combinations of INH+RFP+PZA+EMB, INH+RFP+EMB and INH+EMB. DILI-inducing antipsychotics were chlorpromazine, perphenazine, citalopram, flupenthixol, aripiprazole, clozapine and carbamazepin. DILI-inducing NSAIDs were APAP, celecoxib and ibuprofen. DILI-inducing antineoplastics were methotrexate, nedaplatin, cisplatin, carboplatin, pemetrexed, gefitinib and imatinib. DILI-inducing antithyroids were methimazole and propylthiouracil. DILI-inducing antibiotics were cephalosporins, macrolides, quinolones, nitrofurantoin, ketoconazole, tretinoin and terbinafine. DILI-inducing antihypertensives were amlodipine, captopril and irbesartan. DILI-inducing antidiabetics were metformin, acarbose and glipizide. DILI-inducing anti-HIV drugs were lamivudine + nevirapine + zidovudine combination. Other DILI-inducing drugs included bezafibrate, mifepristone, methylprednisolone and levothyroxine sodium.

Comparison of clinical manifestations between Chinese and Western drug-induced DILI. Comparison of top 3 clinical symptoms (weakness, poor appetite and dark urine) between Chinese and Western drug groups revealed that dark urine symptom was more common in the Chinese drug group. No significant difference was found in biochemical indices such as ALT, AST, ALP, TBiL, PT, PTA and INR between the 2 groups.

Autoantibody-positive rate for Chinese drug-induced DILI. There were 252 patients (59.4\%) with Chinese drug-induced DILI, wherethe top 3 DILI-inducing drugs with clear ingredients were Polygonum multiflorum (17 patients), Tripterygium wilfordii (11 patients) and Sedum aizoon (8 patients). Among all patients with Chinese drug-induced DILI, 143 (56.7\%) were autoantibody-positive, while 109 (43.3\%) were autoantibody-negative. For the Polygonum multiflorum group, 10 patients were autoantibody-positive and 7 patients were autoantibody-negative. For the Tripterygium wilfordii group, 5 patients were autoantibody-positive and 6 patients were autoantibody-negative. And for the Sedum aizoon group, 6 patients were autoantibody-positive and 2 patients were autoantibody-negative (Table IV).

Autoantibody-positive rate for Western drug-induced DILI. There were 172 patients (60.6\%) with Western drug-induced DILI, where the top 5 DILI-inducing drugs with clear ingredients were antituberculotics (72 patients), antipsychotics (27 patients), NSAIDs (17 patients), antineoplastics (13 patients) and antithyroids (11 patients). Among all patients with Western drug-induced DILI, 93 (54.1\%) were autoantibody-positive, while $79(45.9 \%)$ were autoantibody-negative. For the antituberculotics group, 51 patients were autoantibody-positive and 21 patients were autoantibody-negative; for the antipsychotics group, 14 patients were autoantibody-positive and 13 patients were autoantibody-negative. For the NSAIDs group, 11 patients were autoantibody-positive and 5 patients

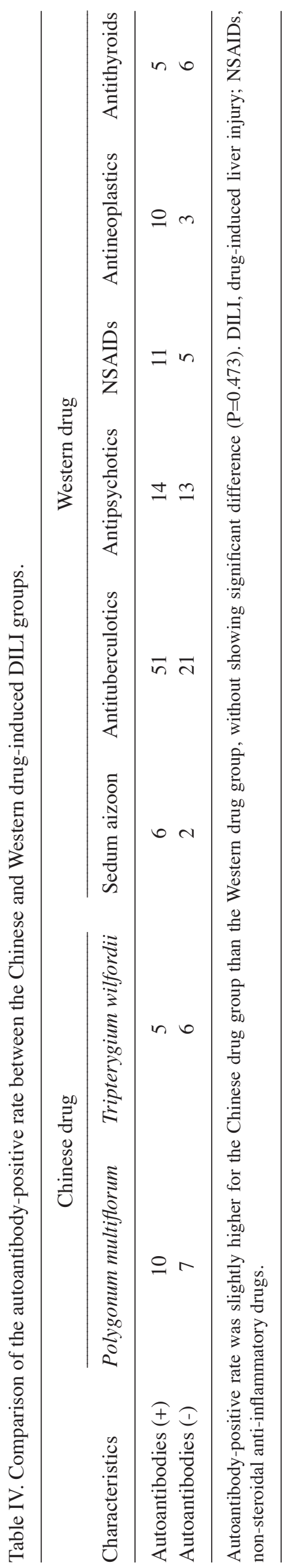


were autoantibody-negative. For the antineoplastics group, 10 patients were autoantibody-positive and 3 patients were autoantibody-negative. For the antithyroids group, 5 patients were autoantibody-positive and 6 patients were autoantibody-negative (Table IV).

Logistic regression analysis of risk factors. One-way ANOVA of possible DILI-related factors i.e., age $\geq 45$ years, chronic alcohol, underlying hepatobiliary diseases, immune dysfunction, hypertension and diabetes, showed that all factors exhibited significant differences in the incidence of DILI $(\mathrm{P}<0.05)$, all of which were DILI-inducing factors. Multivariate analysis was performed selecting these 6 DILI-related factors via logistic regression model, and the results found that the odds ratio (OR) indicated statistical association between the disease and exposure factors, where an OR value $>1$ indicated that the exposure factors were risk factors. Our study demonstrated that DILI risk factors (OR value $>1$ ) included underlying hepatobiliary diseases (6.552), immune dysfunction (6.130), diabetes (3.774), hypertension (2.801), chronic alcohol (2.002) and age $\geq 45$ years (1.838), suggesting that all of these six factors were risk factors for DILI.

Comparison of prognosis between Chinese and Western drug-induced DILI. Cure and improvement were regarded as effective treatment, while non-cure and death were considered ineffective treatment. After comprehensive routine medical treatment, treatment was effective in 241 patients and ineffective in 11 patients in the Chinese drug-induced DILI group, while treatment was effective in 164 patients and ineffective in 8 patients in the Western drug-induced DILI group, showing no significant difference.

\section{Discussion}

Currently, no unified DILI database has been created at home or abroad, nor has the pathogenesis of DILI been fully elucidated. Through statistical analysis of the clinical data, we explored in depth the pathogenesis of DILI and summarized the similarities in DILI-inducing drugs, clinical features and laboratory indices.

Clinical characteristics of the patients treated at our hospital are summarized as follows. The patients mainly presented clinical symptoms such as weakness, poor appetite, yellowed skin, icteric sclera, dark urine, nausea, abdominal discomfort, fever, itchy skin, ascites and rash on admission. But it is worth noting that $10.8 \%$ of the patients showed no obvious clinical symptoms. Clinical symptoms with incidences $\geq 50 \%$ were weakness, poor appetite and dark urine in descending order. Hepatocellular DILI is the type with the highest probability of occurrence. Cholestatic patients had the highest ALP and PTA levels $(\mathrm{P}<0.05)$. Clinical manifestations of DILI lacked specificity compared to other liver diseases, and difference in the drugs used may be the cause of the differing DILI types.

With a male to female ratio of $\sim 1: 1.43$, female DILI patients were higher in number than males, and women are probably at a higher risk of DILI. In our study, patients had an average age of 47.9 years. A study found no significant association of age and gender with DILI occurrence (7), but with increasing age, patients are prone to develop age-related diseases, their organs deteriorate gradually, and hepatic drug metabolizing capacity weakens, thus increasing burden on the liver (8). Body conditions at different ages may be an important reason for differing severity and emergency of DILI. Therefore, on the premise of maintaining blood drug concentration at a therapeutically effective level, the dose and frequency of administration should be appropriately reduced to lower the incidence of DILD.

In this study, the proportion of patients with Chinese drug-induced DILI was $\leq 59.4 \%$. Incidence of Chinese drug-induced DILI has been increasing in recent years; patient misunderstanding of Chinese drugs and limited understanding of drug hepatotoxicity are important contributors to Chinese DILI at present. Lack of efficient drugs for dermatoses, osteoarthritis, gynecopathy and other chronic, obstinate diseases in Western medicine often leads patients to seek TCM treatment, who may even abuse, misuse or chronically use the so-called folk remedies, ancestral recipes and liquor prescriptions. This explains why Chinese drug-induced DILI is mainly concentrated among the population receiving treatment for hair loss, psoriasis, osteoarthritis or receiving health care. Chinese Pharmacopoeia (2010 edition) clearly specifies 72 types of hepatotoxic Chinese drugs, of which common ones include Rheum, Tripterygium wilfordii, Cassia seed, Polygonum multiflorum, Swertia davidi and Aconitum carmichaelii. In this study, the patients' main purposes for taking Chinese drugs were to treat dermatoses, osteoarthrosis and for health care. Compared with Western drugs, Chinese drugs induced more cases of DILI, and caused more frequent dark urine symptom. In our study, the top three frequently DILI-inducing Chinese drugs were found to be Polygonum multiflorum, Tripterygium wilfordii and Sedum aizoon. Analysis of these three herbs found that 17 patients took Polygonum multiflorum for nourishing liver and kiney, blackening beard and hair, strengthening bones and muscles, or treating vertigo, tinnitus, grey hair, hair loss or sore and weak waist and knees, all of whose initial clinical symptoms were weakness and dark urine. The liver injury-inducing mechanism of Polygonum multiflorum may be that the hepatotoxic substances produced during metabolism by anthraquinone derivatives such as rhein and emodin it contains cause hepatocellular lipid peroxidation to induce liver cells necrosis or interfere with hepatocellular uptake and bile secretion while destroying bile salt carrying receptors of the cell membrane, affecting cell membrane $\mathrm{Na}^{+}-\mathrm{K}^{+}$-ATP activity, and damaging the normal structure and metabolic function of liver cells. In this study, 11 patients took Tripterygium wilfordii for treating autoimmune diseases such as rheumatoid arthritis and systemic lupus erythematosus. Their clinical manifestations were abnormal transaminase elevations, accompanied by weakness, poor appetite and icteric sclera symptoms. Liver injury-inducing mechanism of Tripterygium wilfordii may be associated with lipid peroxidation, immunologic injury, excessive hepatocellular apoptosis and P450 enzyme system metabolic abnormalities (9). Hepatic venular occlusive disease (HVOD)-inducing mechanism of Sedum aizoon may be that the metabolites of its major components pyrrolidine alkaloids (PAs), i.e., dehydro-PAs, may act on cellular DNAs and proteins to form DNA polymers, protein polymers, as well as DNA-DNA, DNA-protein polymers, thereby resulting in hepatotoxicity (10). In our study, 8 patients took Sedum aizoon for treatment of traumatic bleeding and traumatic injuries, all of 
whose initial clinical symptoms included evident weakness, poor appetite, dark urine, abdominal distension and pain and ascites. Manifestations of HVOD were present on imaging and pathological examinations, which were degeneration, necrosis and atrophy of liver cells, and extensive occlusion of central lobular veins and hepatic venules.

Current research on TCM toxicology, especially on the hepatotoxicity of Chinese drugs has some limitations. The efficacy and safety of Chinese drugs are still substantially based on experience, which lack support from clinical controlled trials and toxicological studies. DILI-inducing mechanisms of Chinese drugs, which are characterized by complex chemical composition and pharmacological activities, have not yet been fully elucidated. Drug detoxifying and eliminating enzyme systems, differences in single nucleotide polymorphisms of drug transporter genes, direct hepatocellular damage by drugs and their toxic metabolites, or allergic reactions to drugs are all likely to result in liver cell injury. Besides, poor quality of pharmaceutical preparations and crude drugs, differing preparation processes of patent preparations, pesticide residues in medicinal herbs and confusion of varieties (11) are also important causes of liver injury. Moreover, most real-world TCM prescriptions contain multiple herbs, with complex composition and large individual dose discrepancy. Ingredients of some folk remedies are even unclear, which makes it difficult to identify the exact herbs responsible for DILI. All of these pose some challenges to the clinical diagnosis of DILI.

Western drug-induced DILI. The exact mechanisms of Western drug-induced DILI remain unclear. Overall, the mechanisms are substantially the same, which may be classified into the following two types: i) direct hepatotoxic action by drug metabolites (primary compounds); and ii) idiosyncratic liver injury, which is a type of hypersensitivity reaction and the primary mechanism of DILI. Drugs or metabolites can directly damage the liver via lipid peroxidation as free radicals, which can also form complete antigens (drugs or metabolites are haptens) by covalently binding to proteins to stimulate the antibody-dependent cellular cytotoxicity and $\mathrm{T}$ cell hypersensitivity. Direct hepatotoxicity of DILI is predictable, dose-dependent, highly prevalent among individuals, and found mostly in acute injuries. As metabolism is most abundant in the distal hepatic artery region, where the antioxidant and detoxification capacities are the weakest, damaging free radicals firstly invade that area to form zonal hepatocellular necrosis. Idiosyncratic liver injury in DILI is unpredictable, mostly dose-independent, infrequent among individuals, and can lead to hepatocellular injury and (or) cholestasis. It differs from the former in that cellular necrosis within the liver parenchyma is often evenly distributed in each hepatic lobule rather than zonally distributed.

Antituberculotics accounted for $17.0 \%$ of all DILI-inducing drugs, which is the type of Western drugs associated with the highest incidence of DILI. DILI is also one most common cause of discontinued antituberculosis therapy. During antituberculosis therapy, long-term use of antituberculotics is inevitable, thus almost all patients will experience varying degrees of adverse reactions at different frequencies. This study showed that INH, RFP and PZA are the most common antituberculotics inducing DILI, which manifests as abnormal transaminase levels. EMB and streptomycin, on the other hand, induce less DILI, which is mainly related to their metabolic pathways. Active intermediates of acethydrazide, an INH metabolite can covalently bind to intrahepatocellular macromolecules or GSH to damage liver cells, while another metabolite hydrazine can directly peroxidize hepatocytes to induce liver cell injury. RFP combined with INH can provide acetyl groups for the metabolism of INH, thereby resulting in markedly increased acetohydrazide and hydrazine contents, and exacerbate liver cell injury. INH and RFP have greater toxicity when used in combination than used alone. In addition, PZA is a semi-antibiotic, which is often used in combination with INH and RFP. PZA metabolites are likely to be activated to be toxic by the liver enzyme inducing activity of RFP. This also explains why the DILI-inducing antituberculotics in this study were mostly in the forms of INH+RFP+PZA+EMB, $\mathrm{INH}+\mathrm{RFP}+\mathrm{EMB}$ and $\mathrm{INH}+\mathrm{EMB}$ combinations. Liver injury induced by an antituberculotic INH is associated with the individual-specificity of patients. Rapid acetylators contain more $\mathrm{N}$-acetyltransferases, have stronger activity and form more acetyl hydrazine and hydrazine, thus leading to degeneration and necrosis of liver cells. RFP, as an inducer of cytochrome P450, can increase the toxic metabolites of INH and thereby aggravate INH-induced liver injury. Clinical manifestations of antituberculotic-induced DILI are mainly mild to moderate weakness and poor appetite.

The proportion of DILI attributed to antipsychotics ranked second to antituberculotics. Schizophrenic patients need long-term, high-dose or even life-time intake of antipsychotics as the conditions are persistent and easy to relapse, thus greatly increasing the risk of liver injury. This study found that chlorpromazine, a typical antipsychotic, often causes toxic liver injury or obstructive jaundice due to high dosage and prolonged medication, where recovery of liver function after hepatoprotective treatment is undesirable as well. According to some studies $(12,13)$, atypical antipsychotics cause less damage to the liver than the typical antipsychotics; besides, they have less impact on liver function, so the condition quickly returns to normal after treatment or returns to normal spontaneously. Overall, antipsychotic-induced liver injuries are mostly transient ALT elevation without obvious clinical symptoms. The majority of such injuries can easily recover spontaneously with good prognosis. Therefore, antipsychotic-induced DILI normally does not require drug discontinuance, where only hepatoprotective therapy is needed on the basis of monitoring liver function.

In addition, Western drugs associated with high incidence of DILI also included NSAIDs, antineoplastics and antithyroids in the present study. Clinical evidence suggests that NSAIDs have liver toxicity, but the exact incidence of liver injury attributed to each drug is unclear. Only DILIs induced by a few NSAIDs such as aspirin, APAP and benorilate are dose-dependent, and predictable. DILIs induced by most other NSAIDs are dose-independent, and unpredictable. Antineoplastics are also one of the most common drugs responsible for DILI. In this study, common symptoms of antineoplastic-induced DILI included fever, jaundice, rash and liver pain, of which jaundice and liver pain were more common in cholestatic DILI. Major antithyroids responsible for DILI were propylthiouracil and methimazole in this study. Propylthiouracil-induced liver injuries are mainly varying 
degrees of liver cell necroses, which manifest as elevated transaminases, while methimazole-induced liver injuries are mainly intrahepatic cholestasis, which mainly manifests as elevated BiL.

Analysis of risk factors for DILI. There are numerous factors influencing DILI including race $(14,15)$, age, gender, drug type, dose, route of administration, drug combination, genetic factors, diet (16), malnutrition, underlying liver lesions (17), which may all affect patients' susceptibility to DILI. In our study, $38.4 \%$ of patients originally suffered from hepatobiliary diseases, and $55.4 \%$ of patients had underlying diseases. The study further analyzed the risk factors for DILI by logistic regression, which found that the risk of DILI for patients with hepatobiliary diseases was 6.552 times that of patients without underlying hepatobiliary diseases, indicating that hepatobiliary diseases would increase the likelihood of DILI development. Research has confirmed that liver disease can increase the risk of DILI (18). Hepatobiliary diseases can reduce the hepatic ability to decompose, transform and remove drugs, and prolong drug retention in the body, thereby increasing the incidence of DILD. For instance, hepatitis is likely to increase the patients' susceptibility to drugs, which thus increases the incidence of hepatotoxicity.

This study showed that the risk of DILI for diabetic patients was 3.774 times that noted in the non-diabetic patients and the same risk for hypertensive patients was 2.801 times that in the non-hypertensive patients. Both blood pressure and blood glucose are major components of metabolic syndrome associated with abnormal liver function. Metabolic syndrome is associated with abnormal liver function. Patients with metabolic syndrome have significantly higher ALT than those without metabolic syndrome, according to a study (19). For instance, patients with type 2 diabetes often have abnormal lipid metabolism, oxygen stress and lipid peroxidation, abnormal changes in lipid factors, liver iron overload and abnormal accumulation of hepatic glycogen, resulting in great burden on the liver or liver impairment (20). Furthermore, chronic intake of liver metabolized drugs increases the metabolic burden on the liver, which easily leads to liver injury.

Intrahepatic immune cells and their secreted cytokines regulate the hepatic and systemic immune response. Occurrence and progression of DILI are interrelated and interactive with systemic immune dysfunction. Hepatic sinusoidal endothelial cells, lymphocytes, hepatic macrophages and neutrophils play important roles in the immunoregulatory mechanisms of DILI (21). Our study showed that the risk of DILI for patients with immune dysfunction was 6.13 times that noted in the immunocompetent patients. Excessively strong immune function causes allergies or autoimmune diseases, while excessively weak immune function leads to combined immunodeficiency and cancer. Those with allergic constitution are often more sensitive to drugs, more prone to adverse drug reactions such as drug eruption, urticaria and asthma, and have higher incidence of DILI. Patients with combined immunodeficiency or cancer, on the other hand, have increased susceptibility to drug hepatotoxicity.

Heavy drinking can cause or aggravate DILI; the risk can increase by 2-4 times compared to non-drinkers (22). Our study showed that the risk of DILI for chronic drinker patients was 2 times that noted for non-chronic drinker patients. Direct hepatotoxicity of ethanol and indirect hepatotoxicity of its metabolite acetaldehyde result in hepatocellular damage. Drug intake by chronic drinker patients under hepatocellular damage state will increase the incidence of hepatotoxicity.

Advanced age is often a risk factor for DILI. DILI accounts for $\leq 20 \%$ of all liver diseases among China's elderly population; and $40 \%$ of elderly patients hospitalized for acute liver diseases have DILI. Risk of DILI for patients $\geq 45$ years of age is 1.838 times that of the patients $<45$ years of age. Phase I and II drug metabolizing enzyme activities do not change with increasing age $(23,24)$, but various organs deteriorate gradually. Reduced CYP3A removing capacity is associated with renal hypofunction (25), and reduced renal function leads to increased drug accumulation in the liver. In addition, prevalence of cardio-cerebrovascular and other chronic diseases increases with age, which often leads patients to chronically intake various drugs, healthcare products or Chinese medicinal preparations, thus easily causing DILI.

Although DILI is more prevalent among women, the autoantibody-positive rate for DILI was independent of gender, age, type of liver injury and CHILD classification. In this study, the medication time $\geq 30$ day group had a significantly higher autoantibody-positive rate than that noted in the medication time $<30$ day group, suggesting a correlation between the autoantibody-positive rate and medication time, that is, the longer the medication time, the higher the positive rate. Patients with medication time $\geq 30$ days were often chronic DILI patients, who presented high autoantibody positivity. With increasing CHILD score, the autoantibody-positive rate exhibited a gradually increasing trend. DILI with autoantibody positivity may portend severe liver injury outcome. Autoantibody-positive rate was similar among the different clinical types in this study, showing no significant difference. This suggests that drug-induced autoimmune responses can occur in both parenchymal liver cells and bile duct cells. Autoantibody positivity is more prevalent in hepatocellular DILI patients, who have significantly increased ALT, AST and may accompany elevated serum TBiL.

According to the 3-step injury model of DILI, first, drugs and their metabolites directly induce cellular stress, and inhibit intracellular mitochondrial function or activate specific immune response. Subsequently, the initial cellular damage leads to mitochondrial permeability transition, and finally transition of mitochondrial permeability inhibits ATP synthesis leading to necrosis (3). Therefore, combining the three-step DILI injury model with the findings in the present study that both Chinese and Western drugs can cause autoantibody positivity, DILI is still considered attributed to two mechanisms: i) direct hepatotoxicity by drug metabolites (primary compounds); and ii) idiosyncratic liver injury, with probable immune activation. The autoantibody-positive rate was higher in the Chinese drug-induced DILI group than the rated in the Western drug group, but the difference was insignificant, suggesting that the autoantibody-positive rate was independent of drug type. It is thus clear that in addition to the association with patients' idiosyncrasy, idiosyncratic DILI may also be associated with drug-related factors, which is the result of synergistic interactions between drug, host or environmental factors. 
Classic Hy's law provides an important basis for evaluating the prognosis of DILI. When ALT $\geq 3$ the upper limit of normal (ULN) and TBL $>2$ ULN, it generally indicates a poor prognosis, where mortality can reach $10-50 \%$ in the absence of biliary obstruction even after discontinuation of relevant drugs. In the presence of intrahepatic cholestasis and absence of of biliary obstruction and hepatocellular transporter protein dysfunction, continuous TBiL elevation means severe necrosis of liver cells causing obstructed bile excretion pathway, which suggests a poor prognosis. There is no specific treatment for DILI yet, and therapeutic efficacy is still uncertain. In our study, the therapeutic regime was discontinuation of the suspected drugs supplemented by comprehensive anti-inflammatory, detoxifying, choleretic and jaundice-removing medical treatment. Comparison of prognosis found that both the Chinese and Western drug-induced DILI patients achieved good outcome, showing no statistical significance. Thus, regardless of the type of drugs (Chinese or Western drugs) responsible for DILI, even though DILI has no specific treatment yet with uncertain efficacy, timely discontinuation of the suspected drugs and active hepatoprotective treatment will demonstrate some clinical efficacy.

In conclusion, hepatotoxicity of most Chinese and Western drugs and their pathogeneses are unclear at present, nor are there any systematic criteria for monitoring and statistical analysis of DILI. Nevertheless, the present study believes that the pathogenic mechanisms of DILI are substantially the same for Chinese and Western drugs. To lower the incidence of DILI, dose and frequency of administration should be appropriately reduced on the premise of ensuring blood drug concentration.

Compared to other liver diseases, DILI has no specific diagnostic marker and its clinical manifestations are also nonspecific. All patients who manifest weakness, nausea, vomiting, loss of appetite, liver discomfort and other symptoms or demonstrate abnormal liver function during or after intake of drugs suspected of causing liver injury should be given high attention.

For DILI patients, the longer the medication time, the higher the positive rate of autoantibodies. Detection of autoantibodies in patients with abnormal liver function as a result of prolonged medication has significant referential value. Both Chinese and Western drugs can cause autoantibody positivity in DILI. Detection of autoantibodies may have no significant referential value for differentiation between Chinese and Western drug-induced DILI. Nevertheless, Chinese and Western drugs induce DILI via three major pathways, i.e., direct cellular stress response, direct damage to mitochondrial function and stimulation of specific immune response, which may have some association with autoantibody positivity. DILI accompanied by autoantibody positivity may indicate severe liver injury outcome. Autoantibody positivity is more prevalent among hepatocellular DILI patients. Therefore, if patients with prolonged use of hepatotoxic drugs and significantly elevated ALT and AST are tested positive for autoantibodies, it will have certain significance to predicting the outcome of liver injury.

Hepatobiliary diseases, diabetes, hypertension, advanced age, chronic alcohol consumption and immune dysfunction are likely to increase drug susceptibility, and patients with these diseases are prone to liver injury. In clinical practice, the possibility of DILI should be considered firstly for liver dysfunctional patients possessing these risk factors. Thus, faced with complex pathogeneses, prevention of DILI seems particularly important. For patients with a history of drug allergy, chronic medication, advanced age, hepatobiliary diseases or underlying diseases, special attention should be paid to closely monitor the liver and kidney function parameters when using new drugs, and the abnormalities should be managed as early as possible while discontinuing the suspected drugs and administering active hepatoprotective therapy. Patients with a history of DILI should be informed of drugs with the same or similar chemical structures, so as to avoid the recurrence of DILI.

In conclusion, comprehensive clinical analysis of patient clinical features, hepatotoxic drugs, liver function indices, autoantibodies and underlying diseases is of vital and profound significance to the first time diagnosis of DILI, and to the prediction of DILI occurrence and progression, which not only provides direction to the creation of pathogenesis models for various drugs, but also lays a foundation for the future establishment of molecular detection and clinical evaluation platforms for DILI.

\section{References}

1. Larrey D: Drug-induced hepatitis: Epidemiologic, clinical, diagnostic and physiopathologic aspects in 1995. Rev Med Interne 16: 752-758, 1995 (In French).

2. Kaplowitz N: Drug-induced liver injury. Clin Infect Dis 38 (Suppl 2): S44-S48, 2004

3. Russmann S, Jetter A and Kullak-Ublick GA: Pharmacogenetics of drug-induced liver injury. Hepatology 52: 748-761, 2010.

4. McGill MR and Jaeschke H: MicroRNAs as signaling mediators and biomarkers of drug- and chemical-induced liver injury. J Clin Med 4: 1063-1078, 2015.

5. Ward J, Kanchagar C, Veksler-Lublinsky I, Lee RC, McGill MR, Jaeschke H, Curry SC and Ambros VR: Circulating microRNA profiles in human patients with acetaminophen hepatotoxicity or ischemic hepatitis. Proc Natl Acad Sci USA 111: 12169-12174, 2014.

6. Danan G and Benichou C: Causality assessment of adverse reactions to drugs - I. A novel method based on the conclusions of international consensus meetings: Application to drug-induced liver injuries. J Clin Epidemiol 46: 1323-1330, 1993.

7. Lucena MI, Andrade RJ, Kaplowitz N, García-Cortes M, Fernández MC, Romero-Gomez M, Bruguera M, Hallal H, Robles-Diaz M, Rodriguez-González JF, et al; Spanish Group for the Study of Drug-Induced Liver Disease: Phenotypic characterization of idiosyncratic drug-induced liver injury: The influence of age and sex. Hepatology 49: 2001-2009, 2009.

8. Chalasani $\mathrm{N}$ and Björnsson $\mathrm{E}$ : Risk factors for idiosyncratic drug-induced liver injury. Gastroenterology 138: 2246-2259, 2010.

9. Zhu Y, Li YG, Wang JB, Liu SH, Wang LF, Zhao YL, Bai YF, Wang ZX, Li JY and Xiao XH: causes, features, and outcomes of drug-induced liver injury in 69 children from China. Gut Liver 9: 525-533, 2015.

10. Ma X, Peng JH and Hu YY: Chinese Herbal Medicine-induced Liver Injury. J Clin Transl Hepatol 2: 170-175, 2014.

11. Navarro VJ and Senior JR: Drug-related hepatotoxicity. N Engl J Med 354: 731-739, 2006.

12. Daly AK: Pharmacogenomics of adverse drug reactions. Genome Med 5: 5, 2013.

13. Douros A, Bronder E, Andersohn F, Klimpel A, Thomae M, Sarganas G, Kreutz R and Garbe E: Drug-induced liver injury: Results from the hospital-based Berlin case-control surveillance study. Br J Clin Pharmacol 79: 988-999, 2015.

14. Patin E, Harmant C, Kidd KK, Kidd J, Froment A, Mehdi SQ, Sica L, Heyer E and Quintana-Murci L: Sub-Saharan African coding sequence variation and haplotype diversity at the NAT2 gene. Hum Mutat 27: 720, 2006. 
15. Kim SH, Kim SH, Bahn JW, Kim YK, Chang YS, Shin ES, Kim YS, Park JS, Kim BH, Jang IJ, et al: Genetic polymorphisms of drug-metabolizing enzymes and anti-TB drug-induced hepatitis. Pharmacogenomics 10: 1767-1779, 2009.

16. Chen M, Borlak J and Tong W: High lipophilicity and high daily dose of oral medications are associated with significant risk for drug-induced liver injury. Hepatology 58: 388-396, 2013.

17. Maddox JF, Amuzie CJ, Li M, Newport SW, Sparkenbaugh E, Cuff CF, Pestka JJ, Cantor GH, Roth RA and Ganey PE: Bacterial- and viral-induced inflammation increases sensitivity to acetaminophen hepatotoxicity. J Toxicol Environ Health A 73: 58-73, 2010.

18. Russo MW and Watkins PB: Are patients with elevated liver tests at increased risk of drug-induced liver injury? Gastroenterology 126: 1477-1480, 2004.

19. Zamor PJ and Russo MW: Liver function tests and statins. Curr Opin Cardiol 26: 338-341, 2011.

20. Baig NA, Herrine SK and Rubin R: Liver disease and diabetes mellitus. Clin Lab Med 21: 193-207, 2001.

21. Oda S and Yokoi T: Establishment of animal models of drug-induced liver injury and analysis of possible mechanisms. Yakugaku Zasshi 135: 579-588, 2015 (In Japanese).
22. Chinese Society for Tuberculosis and Chinese Medical Association: Recommendations for diagnosis and treatment of anti-tuberculosis drug induced liver injury. Chin J Tuberc Respir Dis 36: 732-736, 2013.

23. He LN, Yang AH, Cui TY, Zhai YR, Zhang FL, Chen JX, Jin CH, Fan YW, Wu ZJ, Wang LL, et al: Reactive metabolite activation by CYP2C19-mediated rhein hepatotoxicity. Xenobiotica 45: 361-372, 2015.

24. Cotreau MM, von Moltke LL and Greenblatt DJ: The influence of age and sex on the clearance of cytochrome P450 3A substrates. Clin Pharmacokinet 44: 33-60, 2005.

25. Lewis JH, Mortensen ME, Zweig S, Fusco MJ, Medoff JR and Belder R; Pravastatin in Chronic Liver Disease Study Investigators: Efficacy and safety of high-dose pravastatin in hypercholesterolemic patients with well-compensated chronic liver disease: Results of a prospective, randomized, double-blind, placebo-controlled, multicenter trial. Hepatology 46: 1453-1463, 2007. 